\title{
Comment on Jang $\mathbf{N}$ et al.: Operative versus nonoperative treatment for acute Achilles tendon rupture: a meta-analysis based on current evidence
}

\author{
Xue-Qiang Wang • Jie-Jiao Zheng • Ying-Hui Hua • \\ Bin Cai
}

Received: 24 May 2012 / Accepted: 5 June 2012 / Published online: 21 June 2012

(C) Springer-Verlag 2012

In International Orthopaedics, Nan Jiang and his colleagues conduct a meta-analysis of operative versus nonoperative treatment for acute Achilles tendon rupture (AATR), who do very well [1]. However, the meta-analysis has brought a few questions to our minds that we would like to note to the authors:

1. Six electronic databases (Medline, Embase, Clinical Ovid, BIOSIS and Cochrane registry of controlled clinical trials) were systematically searched by the authors. However, the authors had not focused specifically or in any detail on the issue of the completeness of the search strategy report for databases. Search strategy report plays an important role in systematic reviews.

2. Most of the patients were middle-aged (average age of 40 years) in the meta-analysis. Although Achilles tendon ruptures occur most frequently in the middle-aged, we want to know whether or not the results of the metaanalysis are suitable for all kinds of people.

X.-Q. Wang · J.-J. Zheng $(\bowtie)$

Department of Sport Rehabilitation, Shanghai University of Sport, 200 Hengren Rd,

Shanghai 200438, China

e-mail: zjjcss@126.com

\section{J.-J. Zheng}

Department of Rehabilitation Medicine,

Huadong Hospital Affiliated to Fudan University,

Shanghai, China

Y.-H. Hua

Department of Sport Medicine,

Huadshan Hospital Affiliated to Fudan University,

Shanghai, China

B. Cai

Department of Rehabilitation Medicine, Affiliated No. 9 People's Hospital, School of Medicine, Shanghai Jiao Tong University,

Shanghai, China
3. The authors should independently extract the following additional data: details of operative and nonoperative treatment. At present, there are different approaches in either operative or nonoperative treatment for AATR, which would bring different results. If possible, we suggest that a meta-analysis of different operative approaches versus different nonoperative treatment for AATR could be conducted.

4. The authors used the modified Jadad scale to assess the risk of bias for all articles, but there were no detail scores for every article. Methodological quality for all articles should be respectively evaluated by two authors; however, the authors did not describe it in the meta-analysis.

5. The result of the meta-analysis showed that operative was superior to nonoperative treatment regarding lower risk of re-rupture $(\mathrm{P}=0.002)$, but we consider that any treatment methods for AATR should not only consider Achilles tendon rupture severity, but also the medical costs and other risks.

6. Publication language was limited to English in the metaanalysis. Therefore, the authors should mention the potential importance of language bias in the limitations of their meta-analysis.

We agree on the following conclusions of the authors: the sample size was not too large to draw any firm conclusions for AATR, and further rigorous, multi-centre RCTs with large sample size need to be carried out.

\section{References}

1. Jiang N, Wang B, Chen A, Dong F, Yu B (2012) Operative versus nonoperative treatment for acute Achilles tendon rupture: a metaanalysis based on current evidence. Int Orthop 36:765-773 